\title{
A Review of Inverted Papilloma at a Tertiary Centre: A Six-Year Experience
}

\author{
Chiun Kian Chai ${ }^{1 *}$, Ing Ping Tang ${ }^{2}$, Narayanan Prepageran ${ }^{1}$ \\ ${ }^{1}$ Department of Otorhinolaryngology, University of Malaya, Kuala Lumpur, Malaysia \\ ${ }^{2}$ Department of Otorhinolaryngology, Faculty of Medicine, University Malaysia Sarawak, Kota Samarahan, Malaysia \\ Email: *chiun_kian@yahoo.com.sg
}

Received December 16, 2012; revised January 12, 2013; accepted January 30, 2013

Copyright (C 2013 Chiun Kian Chai et al. This is an open access article distributed under the Creative Commons Attribution License, which permits unrestricted use, distribution, and reproduction in any medium, provided the original work is properly cited.

\begin{abstract}
Objectives: To evaluate the clinical presentation and treatment outcome of all patients treated for inverted papilloma at the University Malaya Medical Centre, Malaysia. Methods: A retrospective review of 22 patients with inverted papilloma, treated at our centre, from 1st January 2005 to 31st August 2011. The demographic data, clinical features, radiological findings, surgical procedures, recurrent rate and malignant transformation were assessed. Result: The commonest presentation was a unilateral nasal blockage, comprising 77.3\% cases. A total of 19 patients had undergone endoscopic surgery while three patients had open surgery performed. The mean duration of the follow-up period was 30.5 months. Seven (31.7\%) patients were detected of tumour recurrence during the entire course of follow-up. However, throughout the follow-up period, none had demonstrated malignant transformation. Conclusion: Endoscopic medial maxillectomy is a well-accepted approach in treating inverted papilloma. Nevertheless, open surgery still plays an important role in extensive diseases and in cases of malignancy. Unilateral nasal obstruction is the most important symptom. Repeated pre-operative biopsy or perhaps frozen section of intraoperative biopsies is necessary to avoid misdiagnosis. Krouse stage II disease showed the highest recurrent rate of $18.2 \%$. Lifelong follow-up and emphasis on adherence is mandatory for early detection of recurrence and malignant transformation.
\end{abstract}

Keywords: Inverted Papilloma; Medial Maxillectomy; Endoscopic Surgery

\section{Introduction}

Inverted papilloma is an important differential diagnosis of unilateral nasal mass. It is a benign tumour which commonly arises from the lateral nasal wall and rarely from the mucosa of the paranasal sinuses [1]. Many terms have been used to describe inverted papilloma, such as Schneiderian papilloma, transitional cell papilloma, epithelial papilloma, and squamous papillary epithelioma [2]. Histopathologically, it is characterized by invagination of the epithelial growth into the underlying stroma [3].

Inverted papilloma is locally destructive, with a high tendency to recur and is associated with malignant transformation [4]. Hence aggressive surgical approach is always advocated. Since the introduction of endoscopic surgery, the trend of surgical approach has moved away from the traditional techniques, e.g. lateral rhinotomy, Denker procedure and Caldwell-Luc approach, to endoscopic approach. The role of endoscopic surgery in inverted papilloma is now well established and achieves

*Corresponding author. similar tumour recurrence rate comparing to open technique [3,5-7]. Nevertheless, open technique still plays its role in extensive diseases, significant anatomic distortion due to previous surgery or in cases associated with malignancy $[8,9]$.

\section{Materials and Methods}

This is a retrospective study of all patients with inverted papilloma treated in our centre from 1st January 2005 to 31st August 2011. The demographic data, clinical features, surgical procedures and the outcome of these patients were collected from the medical record office and statistically analysed. All the patients underwent rigid nasoendoscopic examination, nasal mass biopsy and computed tomography before operations. Krouse staging system was used to grade the disease (Table 1). Post-operatively, nasoendoscopic examination was carried out in clinic setting to look for recurrence or residual disease. The primary goal of this review is to evaluate the clinical presentation, the management and the outcome of in- 
Table 1. Krouse staging.

\begin{tabular}{cc}
\hline Class & Tumour Extent \\
\hline I & Tumour confined to nasal cavity \\
II & $\begin{array}{c}\text { Tumour limited to ethmoid sinus and medial and superior } \\
\text { portion of maxillary sinus }\end{array}$ \\
III & $\begin{array}{c}\text { Tumor involved the lateral and inferior aspect of maxillary } \\
\text { sinus, or involvement of frontal or sphenoid sinus }\end{array}$ \\
IV & Tumour outside nose and sinuses, and include malignancy
\end{tabular}

verted papilloma at our centre.

\section{Results}

A total of 22 patients were analysed. These consisted of 16 males (72.7\%) and six females (27.3\%) with the age ranging from 32 to 71 years old (mean age of 53.8 years old). These 22 patients included 10 Chinese (45.5\%), seven Indians (31.8\%), four Malays (18.2\%) and one foreigner (4.5\%).

The most frequent symptom encountered was nasal blockage, which consisted of 21 (95.5\%) patients. Out of these 21 patients, 17 (77.3\%) patients presented with unilateral nasal blockage while four (18.2\%) patients presented with bilateral nasal blockage. There were eight (36.4\%) patients presented with rhinorrhoea, six (27.3\%) with anosmia, four (18.2\%) with epistaxis and two (9.1\%) with facial pain (Table 2). The duration of symptoms ranged from one week to 60 months with the mean duration of 14.6 months before these patients presented to our otorhinolaryngology department. Among these 22 patients, five (22.7\%) patients had previous nasal polypeptomy done 7 months to 30 years prior to their first presentation at our clinic.

All the patients had nasoendoscopy performed in the clinic setting. Thirteen (59.1\%) patients had right-sided nasal tumour while nine $(40.9 \%)$ patients had tumour over the left nasal cavity. Biopsy was taken in all these patients, and the histopathological examination showed inverted papilloma in 17 (77.3\%) patients and nasal polyp in five (22.7\%) patients. In our study, tumour staging was done not only based on the classical computer tomography (CT) findings, but also intra-operative findings, according to the Krouse classification. There were three (13.6\%) patients with Krouse stage I disease, eight (36.4\%) patients with stage II disease, 10 (45.5\%) with stage III disease and one (4.5\%) with stage IV disease (Table 3).

The mean waiting time for surgery was 3.1 months with a range of less than one month to six months. A total of 19 (86.4\%) patients underwent endoscopic medial maxillectomy while three (13.6\%) patients had open surgery. For the open surgery cases, one patient had Caldwell Luc approach with endoscopic-assisted tumour excision (Krouse stage III) while two other patients had
Table 2. Clinical presentations of inverted papilloma.

\begin{tabular}{cc}
\hline Symptoms & No. of Patients (\%) \\
\hline Nasal Blockage & $21(95.5)$ \\
Unilateral & $17(77.3)$ \\
Bilateral & $4(18.2)$ \\
Rhinorrhoea & $8(36.4)$ \\
Anosmia & $6(27.3)$ \\
Epistaxis & $4(18.2)$ \\
Facial Pain & $2(9.1)$ \\
\hline
\end{tabular}

Table 3. Number of cases according to Krouse staging with the site of involvement.

\begin{tabular}{cccccc}
\hline \multirow{2}{*}{$\begin{array}{c}\text { Krouse } \\
\text { Stage }\end{array}$} & Nose & $\begin{array}{c}\text { Maxillary } \\
\text { sinus }\end{array}$ & $\begin{array}{c}\text { Frontal } \\
\text { sinus }\end{array}$ & $\begin{array}{c}\text { Sphenoid \& } \\
\text { Base of skull }\end{array}$ & Total \\
\hline I & 3 & 0 & 0 & 0 & 3 \\
II & 0 & 8 & 0 & 0 & 8 \\
III & 0 & 8 & 2 & 0 & 10 \\
IV & 0 & 0 & 0 & 1 & 1 \\
\hline
\end{tabular}

lateral rhinotomy with medial maxillectomy (Krouse stage II). The mean duration of surgery was 102.4 minutes for endoscopic surgery, compared to 165 minutes for open surgery. Post-operatively, two (9.1\%) patients from endoscopic group complained of epiphora and one of them suffered peri-orbital oedema with transient diplopia. The eye symptoms resolved spontaneously after few days. The mean duration of hospital stay was 4.6 days in endoscopic group.

The mean duration of the follow-up period was 30.5 months. A total of 11 (50.0\%) patients had defaulted follow-up after the mean duration of 15.7 months postoperatively. Recurrence was detected in seven (31.7\%) patients and two (9.1\%) of them had recurrence twice. These include four (18.2\%) patients with Krouse stage II disease, two (9.0\%) with Krouse stage III disease and one (4.5\%) with Krouse stage IV disease (Table 4). All these patients had undergone endoscopic medial maxillectomy. Maxillary sinus was found to be the commonest site of recurrence, comprising $44.4 \%$ cases (Table 5). The mean duration of recurrence was 21.4 months with a range of six to 72 months. All recurrences were treated with endoscopic tumour excision. Throughout the entire course follow-up period, none of the patients demonstrated malignant transformation.

\section{Discussion}

In our study, the mean age of patients was 53.8 years old, with male to female ratio of 2.7:1. These were consistent with most of the other studies conducted $[2,4]$. The most common presentation in our study was unilateral nasal 
Table 4. Recurrence according to Krouse stage.

\begin{tabular}{ccc}
\hline \multirow{2}{*}{ Krouse Stage } & \multicolumn{2}{c}{ No. of Patients (\%) } \\
\cline { 2 - 3 } & First Recurrence & Second Recurrence \\
\hline I & 0 & 1 \\
II & $4(18.2)$ & $1(4.5)$ \\
III & $2(9.0)$ & $1(4.5)$ \\
IV & $1(4.5)$ & 0 \\
\hline
\end{tabular}

Table 5. Number of cases according to the site of recurrence.

\begin{tabular}{lcc}
\hline \multirow{2}{*}{ Site of Recurrence } & \multicolumn{2}{c}{ No. of Patients } \\
\cline { 2 - 3 } & First Recurrence & Second Recurrence \\
\hline Nose & 2 & 1 \\
Maxillary sinus & & \\
- Roof & 2 & 0 \\
- Lateral wall & 1 & 0 \\
$\quad$ Anterior wall & 1 & 0 \\
$\quad$ Sphenoid & 1 & 1 \\
\hline
\end{tabular}

blockage (77.3\%), followed by rhinorrhea (36.4\%), anosmia (27.3\%) and epistaxis (18.2\%). In other words, unilateral nasal blockage is the commonest symptom, which demonstrates a similar highest percentage as compared to $64 \%$ to $81 \%$ described in other literatures $[2,8]$.

Clinically it is difficult to differentiate between inverted papilloma and inflammatory polyp since they share similar symptoms and signs. Nevertheless, detection of the unilateral nasal mass should raise the index of suspicion for inverted papilloma [2]. In such circumstances, pre-operative biopsy plays an important role in confirming the diagnosis. In our study, however, five (22.7\%) patients had initial pre-operative biopsies showing inflammatory polyps instead of inverted papilloma. These patients had functional endoscopic sinus surgery (FESS) performed, which included uncinectomy, widening of maxillary antrum, anterior and posterior ethmoidectomy. The diagnosis of inverted papilloma was later established from the histopathological specimens sent from these surgeries, proposing the need of endoscopic medial mexillectomy later for these patients. Therefore, in cases highly suspicious of inverted papilloma, perhaps it is worthwhile to repeat another pre-operative biopsy, or take biopsies intra-operatively and send for frozen section to avoid misdiagnosis.

Nowadays, tumour staging is mostly performed with the combination of CT and MRI findings based on Krouse classification. In Malaysia, MRI of paranasal sinuses are not routinely performed due to its expensive cost and lack in facilities. CT, on the other hand, may manifest some artifacts, increasing its difficulty in differentiating tumours from inflamed mucosa, polyps or retained fluid in the paranasal cavity $[6,10]$. Hence, our study modified Krouse classification by integrating intraoperative findings to the CT findings.

In recent years, the trend of surgery had moved from open surgery to endoscopic approach [2,11]. This phenomenon was demonstrated in our study as well, with 19 patients underwent endoscopic surgery and three patients had open surgery performed. Out of the three, two lateral rhinotomy cases were performed in year 2005, and one was converted to Caldwell Luc surgery from initial endoscopic approach when the tumour involvement at the anterior maxillary wall was found intra-operatively. In view of the high recurrent rate of inverted papilloma and its association with malignancy, aggressive excision is always advocated [3,5]. In our centre, patients diagnosed with inverted papilloma would be offered for medial maxillectomy. Surgical margin is extended up to lacrimal bone anteriorly, sparing the nasolacrimal duct; posteriorly, up to the posterior maxillary wall; superiorly, till the base of the skull and; inferiorly, until reaching the floor of the nose. Inferior turbinate is removed while middle turbinate is preserved.

The recurrent rate of inverted papilloma in our study was $31.7 \%$ or seven out of 22 patients. This figure was slightly higher in comparison with $0 \%$ to $27 \%$ reported in others literatures [2-4]. According to the recurrent rate among our patients in correlation with the Krouse staging, the highest recurrent rate of $18.2 \%$ was demonstrated in Krouse stage II disease, followed by Krouse stage III disease (9.1\%), and Krouse stage IV disease(4.5\%). The second recurrent rate among these recurrent cases, on the other hand, was slightly lower in our study, consisting of $28.6 \%$.

All the recurrent cases in our study occurred in the endoscopic surgery group. Among these patients, two had tumour involvement at frontal sinus, which made endoscopic approach technically difficult for tumour clearance, as endoscopic surgery is possible only if the tumour is confined to the frontal recess or the medial part of frontal sinus.

The advantages of the endoscopic approach is not only limited to the cosmetic aspect, it had also shortened the duration of operation as well as hospital stay $[7,12]$. The mean operative duration was 102.4 minutes for each endoscopic procedure. The period of hospital stay was 4.3 days for endoscopic cases in our study, including the day before the operation and the day of operation. In other words, the period of post-operative hospital stay could be just about two days.

The tumour recurrence happened as early as six months or as long as 72 months with the mean time of 21.4 months. Unfortunately, 11 (50.0\%) patients had defaulted follow-up after a mean period of 15.7 months after surgeries. Thus, the follow-up period is definitely 
inadequate to detect tumour recurrence in this context. Malignancy is another main concern, which makes longterm follow-up necessary, with the risk of malignant transformation ranging from $5 \%$ to $21 \%$ as well as the mean duration of 52 months to develop carcinoma, reported in many other literatures $[3,7,13]$. Currently there are no universal guidelines for follow-up regime after surgery. Nevertheless, some authors had suggested for quarterly follow-up in the first two years, six monthly in the subsequent two years and yearly thereafter [3]. In other words, long-term follow up is mandatory and adherence to follow-up should always be emphasized to all patients [10].

\section{Conclusion}

Endoscopic medial maxillectomy is a well-accepted approach in treating inverted papilloma. Nevertheless, open surgery still plays an important role in extensive diseases and in cases of malignancy. Unilateral nasal obstruction is the most important symptom. Repeated pre-operative biopsy or perhaps a frozen section of intraoperative biopsies is necessary to avoid misdiagnosis. Krouse stage II disease showed the highest recurrent rate of $18.2 \%$. Lifelong follow-up and emphasis on adherence is mandatory for early detection of recurrence and malignant transformation.

\section{REFERENCES}

[1] E. Pasquini, V. Sciarretta, G. Farneti, G. C. Modugno and A. R. Ceroni, "Inverted Papilloma: Report of 89 Cases," American Journal of Otolaryngology, Vol. 25, No. 3, 2004, pp. 178-185. doi:10.1016/j.amjoto.2004.01.004

[2] M. A. Thorp, M. F. Oyarzabal-Amiqo, J. H. du Plessis and S. L. Sellars, "Inverted Papilloma: A Review of 53 Cases,” Laryngoscope, Vol. 111, No. 8, 2001, pp. 14011405. doi:10.1097/00005537-200108000-00016

[3] S. G. Wolfe, R. J. Schlosser, W. E. Bolger, D. C. Lanza and D. W. Kennedy, "Endoscopic and Endoscope-Assisted Resections of Inverted Sinonasal Papillomas," Otolaryngology—Head and Neck Surgery, Vol. 131, No. 3, 2004, pp. 174-179. doi:10.1016/j.otohns.2004.05.011
[4] H. Katori and M. Tsukuda, "Staging of Surgical Approach of Sinonasal Inverted Papilloma," Auris Nasus Larynx, Vol. 32, No. 3, 2005, pp. 257-263. doi:10.1016/j.anl.2005.03.015

[5] R. J. Schlosser, J. C. Mason and C. W. Gross, “Aggressive Endoscopic Resection of Inverted Papilloma: An Update," Otolaryngology_Head and Neck Surgery, Vol. 125, No. 1, 2001, pp. 49-53. doi:10.1067/mhn.2001.116789

[6] T. J. Lee, S. F. Huang, L. A. Lee and C. C. Huang, "Endoscopic Surgery for Recurrent Inverted Papilloma,” Laryngoscope, Vol. 114, No. 1, 2004, pp. 106-112. doi:10.1097/00005537-200401000-00019

[7] J. M. Busquets and P. H. Hwang, "Endoscopic Resection of Sinonasal Inveted Papilloma: A Meta-Analysis,” Otolaryngology—Head and Neck Surgery, Vol. 134, No. 3, 2006, pp. 476-482. doi:10.1016/j.otohns.2005.11.038

[8] W. Lawson and Z. M. Patel, "The Evolution of Management for Inverted Papilloma: An Analysis of 200 Cases," Otolaryngology—Head and Neck Surgery, Vol. 140, No. 3, 2009, pp. 330-335. doi:10.1016/j.otohns.2008.11.010

[9] W. M. Mendenhall, R. W. Hinerman, R. S. Malyapa, J. W. Werning, R. J. Amdur, D. B. Villaret and N. P. Mendenhall, "Inverted Papilloma of Nasal Cavity and Paranasal Sinuses,” American Journal of Clinical Oncology, Vol. 30, No. 5, 2007, pp. 560-563. doi:10.1097/COC.0b013e318064c711

[10] G. Eggers, J. Muhling and S. Hassfeld, "Inverted Papilloma of Paranasal Sinuses," Journal of Cranio-Maxillofacial Surgery, Vol. 35, No. 1, 2007, pp. 21-29. doi:10.1016/j.jcms.2006.10.003

[11] F. Carta, B. Verillaud and P. Herman, "Role of Endoscopic Approach in the Management of Inverted Papilloma," Current Opinion in Otolaryngology \& Head and Neck Surgery, Vol. 19, No. 1, 2011, pp. 21-24. doi:10.1097/MOO.0b013e3283425213

[12] P. Peng and G. Har-El, "Management of Inverted Papillomas of the Nose and Paranasal Sinuses," American Journal of Otolaryngology, Vol. 27, No. 4, 2006, pp. 233237. doi:10.1016/j.amjoto.2005.11.005

[13] C. von Buchwald and P. J. Bradley, "Risk of Malignancy in Inverted Papilloma of the Nose and Paranasal Sinuses," Current Opinion in Otolaryngology \& Head and Neck Surgery, Vol. 15, No. 2, 2007, pp. 95-98. doi:10.1097/MOO.0b013e3280803d9b 